\title{
Probiotic Lactobacilli in Microbiology Research
}

\section{Shyamapada Mandal*}

Department of Zoology, University of Gour Banga, Malda, India

*Corresponding Author: Shyamapada Mandal, Laboratory of Microbiology and Experimental Medicine, Department of Zoology, University of Gour Banga, Malda, West Bengal, India. E-mail: samtropmed@gmail.com

Received: October 04, 2017; Published: December 05, 2017

DOI: 10.31080/ASMI.2018.01.0001

\section{Abbreviations}

CPP: Cumulative Probiotic Potential; CNS: Central Nervous System; GRAS: Generally Recognized as Safe; GIT: Gastrointestinal Tract; LAB: lactic Acid Bacteria; MMP: Microbiotic Medicinal Products; ZDI: Zone Diameter of Inhibition

The mounting emergence of multidrug resistant pathogenic bacteria [1] led to search the non-antibiotic biotherapeutics, including the probiotics, in combating bacterial antibiotic resistance, and to treat the infection $[2,3]$. The probiotics are defined as the live microorganisms which, when administered in adequate amounts, confer a health benefit on the host' [4]. Most of the probiotic microorganisms belong to a group of beneficial bacteria, called LAB, among which the lactobacilli (member of the genus Lactobacillus) strains hold significant possession in making them the candidates for probiotics, in terms of their ability to survive the human GIT, and to adhere to the mucosa, for functionality.

The probiotics are incorporated in yogurts, cheese and fermented milk, or accessible as nutritional enhancements in the form of dried products, and the acceptability of probiotic usage has improved radically during the last decades, with an extensive practice in clinical care as well as among the healthy persons desiring to espouse healthy gut microbiota [5]. The probiotic lactobacilli have been confirmed to show therapeutic effects against various pathologies, in preventing metabolic diseases, and on the CNS by reducing the inflammation of intestine [6]. Also, they reduce the risk of pathogen colonization [7], and impact the gut-brain axis, as well [6].

The adaptation of the probiotic strains involves the human food cycle: soil to raw materials, to fermented foods, to human intestine, to feces, and to the soil [8,9]. Essentially, the adaptation of lactobacilli to two dissimilar environmental condition: the human intestine and the extra-intestinal environment, such as food, exerting varied selective pressure related to their growth rate and carbohydrate metabolism measured them as probiotics. Such microorganisms are gram-positive non-spore forming rod, negative to catalase and oxidase production, and are either homofermentative producing lactic acid, or heterofermentative producing $\mathrm{CO}_{2}$ and ethanol along with lactic acid, and have been conferred the GRAS status [10].

The functional features of probiotic lactobacilli are strain specific, and all the probiotic strains are distinctive and dissimilar, the same property for one cannot be extrapolated to that of the other individual strains, even from the same niches, and, therefore, their characteristics are necessitated to be defined well [11]. The sustainability of the strains in the unavoidable biological barriers on ingestion: the acidic state of the stomach and to the bile in the intestine, has been measured vital to verify their prime functionality; therefore, the tolerance to such barriers might explain their poten- tiality to meet the selection criteria of being probiotics [12]. Some of the Lactobacillus species, especially L. gasseri and L. reuteri, have been considered as true commensals of GIT, while other species, such as L. plantarum, L. rhamnosus and L. paracasei, appear to be the transient passengers [13]. Research showed that the selection of new probiotics, intended to be used for health benefits in humans, include initial isolation of the microorganisms that must meet important probiotic qualifications, such as the safety features (being non-pathogenic and not being able to transfer any antibiotic resistance genes to other bacteria), tolerance property (being temperature, acid, $\mathrm{NaCl}$ and bile salt tolerant), antagonistic property (capable to act against bacterial pathogens in the gastrointestinal tract), and finally the accurate identification of the probiotic species and strains.

The appropriate in vitro tests have been adopted to select strains, based on their ability to survive transit through the different compartments of the GIT [14]. Acid tolerance (low-pH) tests are among the simplest tests that can be performed, allowing the routine screening of large numbers of strains. Tolerance to bile salt concentrations between $0.15 \%$ and $0.5 \%$ has been recommended for probiotics, which is in the range of the physiological concentrations met in the GIT [15]. Based upon the facts mentioned above, it has been demonstrated that the functional requirements of probiotics include tolerance to gastric juice and intestinal bile, persistence in the human GIT, antagonistic activity toward bacterial pathogens: Helicobacter pylori, Salmonella spp., Listeria monocytogenes, and Clostridium difficile, and the capacity to stabilize and modulate the intestinal microbiota [16]. The cross-protection against various physical and chemical stressors has been documented; such as the pre-exposure to bile facilitate defense against heat stress, and pre-exposure to sodium chloride secures lactobacilli against bile and heat stress [17]. The LAB, mostly the lactobacilli, possess the capacity to act against pathogenic bacteria by producing antimicrobials, such as $\mathrm{H}_{2} \mathrm{O}_{2}$, organic acids (chiefly, lactic acid) and bacteriocin, by competing for nutrients and binding sites, or by reducing the spread within the colonized body [18], and thus, acquire the enviable property of probiotic potentiality and a sustainable substitute to the conventionally used synthetic antibiotics, and the capacity to maintain and influence the composition of the resilient intestinal microbiota for healthy effect [19].

The probiotic lactobacilli must be safe, and regarding the safety profiling of the strains, two major virulence factors [3]: hemolysis and gelatin hydrolysis, are related to the bacterial pathogenicity, and hence, the in vitro tests for hemolytic activity and gelatinase activity be considered, in order to validate the microorganisms incompetency to cause hemolysis and gelatin liquefaction in host body. In addition, the lack of acquisition of antibiotic resistance is a vital criterion for safety evaluation of lactobacilli to be used as probiotics, since the acquisition of such resistances and their transfer- 
ability to the pathogenic bacteria are of grave concern. Thus, the probiotic lactobacilli are required to be sensitive to antibiotics so as to be incompetent in disseminating the resistance property to other pathogenic bacteria in the shared niches. Also, the antibiotic resistance in probiotic lactobacilli might be the required probiotic feature for survival in the presence of antibiotic(s) co-administered, but the antibiotic resistance among them should be innate and nontransferable [20], and that any given probiotic strain is not at significant risk regarding transferable antibiotic resistance [21].

The probiotic lactobacilli are mostly of milk or milk-product origin, and most of the probiotic foods available in the markets worldwide are milk-based; currently, however, the nondairy based preparation of functional foods containing microorganisms with probiotic potentiality have increased demand due to high milk cholesterol content, potential milk-protein allergens [22] and lactose intolerance in humans, and thus, the nondairy fermented foods represent an advantage in the search for new probiotic strains. Consequence to this, preparation of nondairy probiotic foods (coconut milk, fruit drinks, soy products and cereal based products) have been increased. The studies have shown cereal based probiotic products too, using oat, wheat, and barley with acceptable live cell counts of probiotics. The species of the genus Lactobacillus: L. plantarum, $L$. fermentum and L. Salivarius, from fermented fruits and vegetables had broad antibacterial spectrum (ZDI: 26 - $28 \mathrm{~mm}$ ), against foodborne bacterial pathogens [23]. The availability of commercial probiotics (medical as well as food grade) is emerging, and those in our part of the globe are not less. Nevertheless (as the scientific report on naturally available as well as commercially available probiotic lactobacilli is meager in many parts of the globe), extensive studies on various probiotics strains (native as well as commercial) are required to get a safe and healthy probiotic effect, in conformity with the 'fit for human consumption' protocols [24].

Therefore, the probiotic bacteria, which predominantly belong to lactobacilli, offer exhilarating prospects in medicine because of their capacity to induce a range of beneficial health effects. However, accurate identification of native probiotic strains to the species level is crucial for safety evaluation, and a combination of phenotypic tests and molecular typing, such as 16S rRNA gene sequencing, can confirm the identity of lactobacilli species [22]. Finally, the CPP of the native lactobacilli strains, isolated from locally available sources, is required to be measured as an enhanced criterion for probiotic confirmation [3]. Overall, the LAB, including lactobacilli, from irrespective of sources, cannot be specified as probiotics until and unless their viability, safety and functionality are characterized [25]. However, the scientists around the world must look for the development and expansion of next generation probiotics and MMPs for prophylactic as well as therapeutic strategies [6].

\section{Bibliography}

1. Mandal S. "Can over-the-counter antibiotics coerce people for self-medication with antibiotics?" Asian Pacific Journal of Tropical Disease 5.1 (2015): S184-S186.

2. Halder D., et al. "Antibacterial potentiality of commercially available probiotic lactobacilli and curd lactobacilli strains, alone and in combination, against human pathogenic bacteria". Translational Biomedicine 7 (2016): 1-7.

3. Halder D., et al. "Indigenous probiotic Lactobacillus isolates presenting antibiotic like activity against human pathogenic bacteria". Biomedicines 5.2 (2017): E31.

4. Hill C., et al. "Expert consensus document: the international scientific association for probiotics and prebiotics consensus statement on the scope and appropriate use of the term probiotic". Nature Reviews Gastroenterology and Hepatology 11.8 (2014): 506-514.

5. Islam SU. "Clinical uses of probiotics”. Medicine 95.5 (2016): e2658.

6. El Hage R., et al. "Emerging trends in "smart probiotics": functional consideration for the development of novel health and industrial applications". Frontiers in Microbiology 8 (2017): 1889.

7. Goldenberg JZ, et al. "Probiotics for the prevention of Clostridium difficile-associated diarrhea in adults and children". Cochrane Database of Systematic Review 5 (2013): CD006095.

8. Zheng J., et al. "Comparative genomics Lactobacillus reuteri from sourdough reveals adaptation of an intestinal symbiont to food fermentations". Scientific Reports 5 (2015): 18234.

9. Nuraida L. "A review: Health promoting lactic acid bacteria in traditional Indonesian fermented foods". Food Science and Human Wellness 4.2 (2015): 47-55.

10. Dunne C, et al. "In vitro selection criteria for probiotic bacteria of human origin: correlation with in vivo findings". American Journal of Clinical Nutrition 73.2 (2001): 386S$392 S$.

11. van Bokhorst-van de Veen H., et al. "Congruent strain specific intestinal persistence of Lactobacillus plantarum in an intestine- mimicking in vitro system and in human volunteers". Plos One 7.9 (2012): e44588.

12. Halder D., et al. "Curd lactobacilli with probiotic potentiality". Translational Biomedicine 6 (2015): 1-6.

13. Vesa TP., et al. "Pharmacokinetics of Lactobacillus plantarum NCIMB 8826, Lactobacillus fermentum KLD, and Lactococcus lactis MG 1363 in the human gastrointestinal tract". Alimentary Pharmacology and Therapeutics 14.6 (2000): 823-828. 
14. FAO and WHO. Guidelines for the Evaluation of Probiotics in Food: Report of a Joint FAO/WHO Working Group on Drafting Guidelines for the Evaluation of Probiotics in Food and Agriculture Organization World Health Organization: London, ON, Canada (2002).

15. Gorbach SL., et al. "Nutrition and the gastrointestinal microflora”. Nutrition Reviews 50.12 (1992): 378-381.

16. Swain MR., et al. "Fermented fruits and vegetables of Asia: a potential source of probiotics". Biotechnology Research International (2014).

17. Kim WSL., et al. "Assessment of stress response of the probiotic Lactobacillus acidophilus". Current Microbiology 43.5 (2001): 346-350.

18. Di Cerbo A., et al. "Mechanisms and therapeutic effectiveness of lactobacilli". Journal of Clinical Pathology 69.3 (2016): 187-203.

19. Abdel-Daim A., et al. "Antagonistic activity of Lactobacillus isolates against Salmonella typhi in vitro". Biomed Research International (2013).

20. Jose NM., et al. "Comparison of microbiological and probiotic characteristics of lactobacilli isolates from dairy food products and animal rumen contents". Microorganisms 3.2 (2015): 198-212.

21. NK Ganguly., et al. "ICMR-DBT guidelines for evaluation of probiotics in food". Indian Journal of Medical Research 134.1 (2011): 22-25.

22. Sanders ME., et al. "Safety assessment of probiotics for human use". Gut Microbes 1.3 (2010): 164-185.

23. Manzoor A., et al. "Efficacy of locally isolated lactic acid bacteria against antibiotic-resistant uropathogens". Jundishapur Journal Microbiology 9.1 (2016): e18952.

24. Fijan S. "Microorganisms with claimed probiotic properties: an overview of recent literature". International Journal of Environment Research and Public Health 11.5 (2014): 47454767.

25. Sanders ME. "Probiotics: definition, sources, selection, and uses". Clinical Infectious Diseases 46.2 (2008): S58-S61.

Volume 1 Issue 1 January 2018

(C) All rights are reserved by Shyamapada Mandal. 